\title{
Can IGF-I be used as a Metabolic Biomarker in Professional Volleyball Players during a Training Season?
}

\author{
Marcel Frezza Pisa ${ }^{1 *}$, Enrico Fuini Puggina ${ }^{1,2}$, Eike Bianchi Kohama ${ }^{1}$, Marilia Roque Mansur ${ }^{1}$, Rodrigo \\ Custodio $^{3}$, Carlos Eduardo Martinelli $\mathrm{Jr}^{3}$ and Hugo Tourinho Filho ${ }^{1}$ \\ ${ }^{1}$ School of Physical Education and Sport of Ribeirao Preto, University of Sao Paulo, Ribeirao Preto, Brazil \\ ${ }^{2}$ Post-graduate Program in Rehabilitation and Functional Performance, Ribeirao Preto Medical School, University of Sao \\ Paulo, Ribeirao Preto, Brazil \\ ${ }^{3}$ Department of Pediatrics, Ribeirao Preto Medical School, Universityof Sao Paulo, Ribeirao Preto, Brazil
}

\begin{abstract}
The aim of this study was to investigate the kinetics of serum IGF-I and IGF binding protein (IGFBP)-3 on volleyball athletes during a training season and their relation to the performance in physical tests. Ten adult male volleyball professional players were analysed at baseline (A1), during (A2), and at the end (A3) of 17 weeks of training. Anthropometric data and power of lower limbs in the squat jump, counter movement jump, $40 \mathrm{~cm}$ drop jump (DJ40) were analysed. Serum IGF-I and IGFBP-3 concentrations were collected before and after a standardized training session at A1, A2 and A3. Statistical analyses were performed using ANOVA test and $\triangle \%$. There was no statistically significant difference for the anthropometric measures of Total Body Mass (TBM), \%Body Fat (\%BF), Lean Mass (LM), and Fat Mass (FM) during the training season. The power of lower limbs results showed a linear increase with statistical difference $(p<0.05)$ in the DJ40 at A3. The training session had no influence on the IGF-I and IGFBP-3 concentrations. There was no statistically significant difference between the $\mathrm{A} 1, \mathrm{~A} 2$ and $\mathrm{A} 3$ moments during the training season, although $\Delta \%$ demonstrated an upward trend in serum IGF-I at A3. This higher IGF-I concentration happened together with higher jump height results at A3, indicating potential role between fitness and IGF-I. However, the IGF-I did not prove to be an adequate marker of training status in professional volleyball players during a training season.
\end{abstract}

\section{Keywords}

IGF-I, IGFBP-3, Training season, Metabolic biomarkers, Volleyball

\section{Introduction}

The GH/IGF-I axis (growth hormone - insulin-like growth factor-I) is responsible for somatic tissue growth in several species, including humans. GH is a hormone with anabolic functions, acting on the growth of many tissues (e.g. muscles and bones) and metabolic alterations in the flow of synthesis and degradation of nutrients such as glucose and lipids. An anabolic function of GH occurs through IGF-I. This growth factor is produced mainly in the liver via GH stimulation, but also in many other organs and tissues. Therefore, IGF-I can perform its endocrine, autocrine, and paracrine functions [1].

Evidence shows that the practice of physical exercise during childhood, adolescence, and adulthood may influence the growth and development of muscle mass and bone mass as exercise is closely linked to anabolic function, stimulating the secretion and action of the GH/IGF-I axis [1]. On the other hand, there are some studies that observed decreased $\mathrm{GH}$ and IGF-I concentrations after conducting training programs. The reduction in GH and IGF-I levels may be related toa cata- bolic state, which is expected to reduce physical capacity and body mass [2-4].

According to Rarick, et al. [5] the serum IGF-I concentrations may indicate that there is a close relationship between the IGF-I-induced changes in a short training period and an up regulation caused by a longer training period. It is speculated that the GH/IGF-I axis may present biphasic behavior throughout a training season that can be influenced by the

* Corresponding author: Marcel Frezza Pisa, School of Physical Education and Sport of Ribeirao Preto, University of Sao Paulo, Ribeirao Preto, Av. Bandeirantes, 3900 Monte Alegre, Ribeirao Preto, SP, Brazil, Zip Code: 14040-900, Tel: +5516-99243-5678

Accepted: May 09, 2020

Published online: May 11, 2020

Citation: Pisa MF, Puggina EF, Kohama EB, et al. (2020) Can IGF-I be used as a Metabolic Biomarker in Professional Volleyball Players during a Training Season?. Arch Sports Med 4(1):192197 
training content, with a phase marked by a catabolic state followed by an anabolic state $[3,6]$.

There are few studies about the kinetic of anabolic mediators during a training season. According to Nemet, et al. [7], what causes the reduction in IGF-I during periods of intense training or the optimal increase during tapering phase (reduced training intensity) remains unknown. Nevertheless, the inability to increase circulating IGF-I levels before a target competition should be an alarm signal for both, the athlete and the coach, that the general condition of the athlete could not be ideal. It is still questionable whether the responses of IGF-I and IGF binding protein (IGFBP)-3 levels after acute training sessions differ from the responses observed during a training season, through the different periods of athletic preparation, as well as the influence of possible variations between different sports and planning forms $[3,8]$.

Therefore, we hypothesized that concentrations of these hormones, obtained at different time points of preparation during a training season adult volleyball players, would change related to physical performance of the athletes, and could serve as a training status marker. In addition, monitoring these variables may serve as a metabolic indicator to increase or decrease the training intensity.

Thus, this study aimed to investigate the kinetics of serum IGF-I and IGF-3 on volleyball athletes during a training season and their relation to the performance in physical tests.

\section{Materials and Methods}

The procedures performed in this study were approved by the Research Ethics Committee of the School of Physical Education and Sports, Ribeirao Preto, University of Sao Paulo, being identified by the General Certificate of Appreciation for Ethics number (CAAE) 38761314.5.0000.5659.

\section{Sample}

After being informed verbally and in writing about the procedures to be adopted in the research through the Informed Consent, 10 male athletes with a mean age of $24.6 \pm 3.47$ years met the requirements and participated in the study. The athletes fulfilled a routine of 14 hours and 30 minutes of weekly training from Monday to Friday. They were involved in the Pro-Volleyball Association (APV) championship and the "Regional Games", achieving $1^{\text {st }}$ place (Nov/16) and $2^{\text {nd }}$ place (Jul/16) respectively.

\section{Design study}

The team's follow-up period occurred between the months of March to July. The data were collected at 3 different time points; the first being (A1) two weeks after the start of training, the second (A2) seven weeks after the first sample, and the third (A3) eight weeks after the second samplein the week before the target competition.

The contact platform Jump System Pro (Cefise, Nova Odessa, Brazil) was used connected to a computer with specific software (Jump System 1.0) to determine the jump height and power. The jump tests were the squat jump (SJ), counter movement jump (CMJ) [9], and $40 \mathrm{~cm}$ drop jump (DJ40) [10].
To determine relative power $(\mathrm{Pt} / \mathrm{Kg})$ of the lower limbs the equation proposed by Bosco [11] was used.

Blood samples were collected 30 minutes before and 30 minutes after the training session. The training sessions consisted on average of $\mathbf{2 7 . 7}$ minutes of warm up, which was carried out using stretching of the upper and lower limbs, varied displacements, drills for upper limbs with the ball, technical drills, attack-defense exercises, service and reception, and attacks over the net. The main part of the training consisted on average of 65.3 minutes of action, and was characterized by collective training playing three volleyball sets, similar to a match in competitive conditions. All athletes involved in the research participated fully in all training sessions.

\section{Anthropometric measures}

Height was measured using a portable stadiometer (Avanutribrand) and all procedures were performed according to the Anthropometry Manual [12]; total body mass (TBM) was obtained using a digital scale, (EKS brand, model SI 9805). To determine the body fat percentage (\%BF), the equation of Evans [13] was used. In total three skinfold measurement were taken with a Cescorf adipometer, following the Guedes protocol [14].

\section{Blood collection}

The collection of venous blood was performed via puncture of a blood vessel on the surface of the forearm by a nurse in the training center. Before and after the training session $10 \mathrm{ml}$ were collected into tubes without anticoagulant. The samples were stored in ice at $0-4{ }^{\circ} \mathrm{C}$ and centrifuged at 1,200 $\mathrm{rpm}$ at the same temperature for 15 minutes to obtain the serum, the samples were then aliquoted and stored in $1.5 \mathrm{~mL}$ Eppendorf tubes at $-80^{\circ} \mathrm{C}$ for further hormone analysis.

\section{Determination of serum IGF-I, IGFBP3}

Serum concentrations of IGF-I and IGFBP-3 (Immulite 2000, Siemens, Los Angeles, CA, USA) were determined by specific immunoassays using commercial kits. All samples were analysed in duplicate within the same assay. The intra-assay variations were $2.4 \%$ for IGF-land $2.3 \%$ for IGFBP-3. Assay sensitivity was $5 \mathrm{ng} / \mathrm{ml}$ for IGF-I and $0.1 \mathrm{mg} / \mathrm{l}$ for IGFBP-3.

\section{Diet recall}

In order to analyze the ingestion at the three collection time points, a 24-hour diet recall (R24h), led by a nutritionist was used. Once collected, the information was analyzed by the DietPro Lite program (DietPro, Viçosa, Brazil) and the total amount of carbohydrates, lipids, proteins, and calories ingested by the athletes were determined according to the American Dietetic Association and Dietary Reference Intakes (DRIs).

\section{Statistical treatment}

Statistical procedures were performed using IBM SPSS Statistics software, version 20.0 (IBM Corporation ${ }^{\circ}$ ). The analysis of variance (ANOVA) for repeated measures with one and two factors was used, adopting a significance level of $p \leq 0.05$, 
Citation: Pisa MF, Puggina EF, Kohama EB, et al. (2020) Can IGF-I be used as a Metabolic Biomarker in Professional Volleyball Players during a Training Season?. Arch Sports Med 4(1):192-197

Table 1: Height, TMB, \%BF, LM and FM (mean \pm SD) throughout the training season in volleyball players $(n=10)$ and relative changes.

\begin{tabular}{|l|l|l|l|l|}
\hline Time points & A1 & A2 & A3 & வ\% \\
\hline Height $(\mathrm{m})$ & $1.86 \pm 0.08$ & $1.86 \pm 0.08$ & $1.86 \pm 0.07$ & 0 \\
\hline TBM $(\mathrm{Kg})$ & $81.37 \pm 12.91$ & $81.17 \pm 8.99$ & $77.75 \pm 9.90$ & -4.44 \\
\hline \%BF & $9.16 \pm 3.54$ & $9.65 \pm 3.62$ & $8.68 \pm 3.01$ & -5.24 \\
\hline LM (Kg) & $73.70 \pm 10.56$ & $73.30 \pm 8.29$ & $70.89 \pm 8.51$ & -3.81 \\
\hline FM (Kg) & $7.67 \pm 3.74$ & $7.87 \pm 3.29$ & $6.85 \pm 2.82$ & -10.69 \\
\hline
\end{tabular}

Table 2: IGF-I and IGFBP-3 concentrations (mean \pm SD) in volleyball players $(n=10)$ before and after (pre $\times$ post) standardized training throughout the training season and relative changes.

\begin{tabular}{|c|c|c|c|c|}
\hline & \multirow[b]{2}{*}{ A1 } & \multicolumn{2}{|l|}{ Time points } & \multirow[b]{2}{*}{$\Delta \%$} \\
\hline & & A2 & A3 & \\
\hline IGF-I pre & $218 \pm 97.75$ & $215 \pm 91.76$ & $236 \pm 100.20$ & 8.35 \\
\hline IGF-I post & $216 \pm 96.51$ & $219 \pm 93.44$ & $234 \pm 89.00$ & 8.24 \\
\hline IGFBP-3 pre & $3.76 \pm 0.76$ & $3.66 \pm 0.95$ & $3.80 \pm 0.80$ & 1.06 \\
\hline IGFBP-3 post & $3.79 \pm 0.99$ & $3.73 \pm 1.06$ & $3.82 \pm 0.85$ & 0.79 \\
\hline
\end{tabular}

Table 3: SJ, CMJ, and DJ40 max height and Pw/kg in the SJ and CMJ (mean \pm SD) throughout the training season in volleyball players $(n=10)$ and relative changes.

\begin{tabular}{|c|c|c|c|c|}
\hline & \multicolumn{4}{|l|}{ Time points } \\
\hline & A1 & A2 & A3 & $\Delta \%$ \\
\hline $\mathrm{SJ}(\mathrm{cm})$ & $43.00 \pm 6.99$ & $42.39 \pm 5.44$ & $44.60 \pm 6.36$ & 3.72 \\
\hline $\mathrm{CMJ}(\mathrm{cm})$ & $46.32 \pm 6.65$ & $46.61 \pm 5.23$ & $47.99 \pm 5.48$ & 3.60 \\
\hline DJ40 (cm) & $40.98 \pm 6.37$ & $42.15 \pm 4.66$ & $45.28 \pm 5.84^{*}$ & 10.49 \\
\hline Pt/kg SJ (W) & $52.04 \pm 5.50$ & $51.84 \pm 4.19$ & $53.63 \pm 4.89$ & 3.05 \\
\hline $\mathrm{Pt} / \mathrm{Kg} \mathrm{CMJ}(\mathrm{W})$ & $53.70 \pm 4.36$ & $54.05 \pm 3.30$ & $55.07 \pm 3.39$ & 2.55 \\
\hline
\end{tabular}

$\mathrm{P} \leq 0.05 *$ comparison between time points A1-A3.

and when necessary, the post-hoc Bonferroni test. The normality of the data was certified using the Shapiro-Wilk test and homoscedasticity by the Levene test.

The Pearson correlation test was used to verify possible correlations between the IGF-I concentrations with the results of the motor tests at each collection time point. Relative responses are presented as e.g. $\Delta \%=\left((A 3-A 1) / A 1^{*} 100\right)$.

\section{Results}

\section{Anthropometry}

ANOVA revealed no statistically significant differences between the three collection time points during the season for the anthropometric measures of TBM, \%BF, LM, and FM. When $\triangle \%$ was observed, TBM, \%BF, LM and FM demonstrated a downward trend between the collection times with lower values at $A 3$ (Table 1).

\section{IGF-I and IGFBP-3}

ANOVA revealed no significant differences between pre-training and post-training IGF-I concentrations (acute effect) - IGF-I pre-training (Ax.1) and IGF-I post-training (Ax.2); nor were any observed in the IGFBP-3 concentrations pre and post-training - IGFBP-3 pre-training (Ax.1), and IGFBP-3 post-training (Ax.2). In addition, no interaction was observed between different time points during training and during the season phases (chronic) in the IGF-I and IGFBP-3 concentrations. An upward trend was observed in $\Delta \%$ levels of IGF-I before and after training was found between A1-A3. For IGFBP-3 pre and post training levels, $\Delta \%$ was discreetly elevated between the time points A1-A3 (Table 2).

\section{Vertical jump tests and power of lower limbs}

ANOVA revealed statistically significant differences in the DJ40 test results at $A 3$ compared to $A 1[F=4.50 ; p=0.03$, and $\Delta \%$ showed a considerable change between A1-A3. No differences were found for the jump height values recorded in the power test of the lower limbs in the SJ and CMJ. However, these variables presented a trend to increase between time points A1-A3, which can be an important result considering that the subjects were trained athletes.

There was no significant difference in relative power (Pw/ $\mathrm{kg}$ ) results measured in watts $(\mathrm{W})$ in the $\mathrm{SJ}$ and $\mathrm{CMJ}$, with $\Delta \%$ revealing a quite small difference (Table 3 ).

\section{Correlation between IGF-I concentrations and vertical jump test}

The Pearson correlation test showed that the best cor- 
relation results for each jump test were found at $A 3$. There was a greater increase in IGF-I concentrations and the best results of the three jump tests (SJ, CMJ, and DJ40) (Table 4).

The analysis of the IGF-I and IGFBP-3 concentrations throughout the training season showed no statistically significant differences, however the $\Delta \%$ showed a trend to increase at A3 for IGF-I. Based on these data, it may be reasonable to suggest that the IGF-I concentrations were correlated $(r=$ 0.48 ) with the physical performance of the athletes, since at A3 both IGF-I values and jump height (DJ40) presented their highest values (Figure 1). This fact coincided with the achievement of second place in the competition in which the team participated one week after the tests.

\section{Diet recall}

In the present study, the results of the diet recall analysis were similar at the three time points of evaluation (Table 5).

\section{Discussion}

In the present study, the results of IGF-I and IGFBP-3 concentrations showed no influence of the training session, pos-

Table 4: Pearson correlation between IGF-I concentrations pre-training and vertical jump tests.

\begin{tabular}{|l|l|l|}
\hline IGF-I A1.1 $\times$ SJ A1 & $\begin{array}{l}\text { IGF-I A2.1 } \times \text { SJ A2 } \\
r=0.17\end{array}$ & $\begin{array}{l}\text { IGF-I SJ A3.1 } \times \text { A3 } \\
r=0.34\end{array}$ \\
\hline $\begin{array}{l}\text { IGF-I A1.1 } \times \text { CMJ A1 } \\
r=0.23\end{array}$ & $\begin{array}{l}\text { IGF-I A2.1 } \times \text { CMJ A2 } \\
r=0.17\end{array}$ & $\begin{array}{l}\text { IGF-I A3.1 } \times \text { CMJ A3 } \\
r=0.28\end{array}$ \\
\hline $\begin{array}{l}\text { IGF-I A1.1 } \times \text { DJ40 A1 } \\
r=0.39\end{array}$ & $\begin{array}{l}\text { IGF-I A2.1 } \times \text { DJ40 A2 } \\
r=0.19\end{array}$ & $\begin{array}{l}\text { IGF-I A3.1 } \times \text { DJ40 A3 } \\
r=0.48\end{array}$ \\
\hline
\end{tabular}

sibly indicating that the reality of playing three volleyball sets did not reach the intensity and ideal capacity to cause concentration changes. Eliakin, et al. [15] explained that increases in IGF-I concentrations were associated with above maximum and short-termintensities, and that possibly, volleyball training sessions do not reach this intensity. According to our data, the volleyball training sessions did not cause significant changes in the IGF-I and IGFBP-3 concentrations. This information is supported by Kraemer, et al. [16] who explained that above maximum efforts with a duration of 90 seconds were able to increase IGF-I concentrations.

The results of IGF-I and IGFBP-3 concentrations before and after the training sessions were consistent with data from other studies that analyzed these hormones in male [17], female [18], and male and female [19] volleyball athletes. The researchers suggest that the responses of the IGF-I and IGFBP-3 concentrations, and the relationship with the cortisol and IL- 6 responses promote changes in anabolic/catabolic/ inflammatory hormonal balance and can be used in various team sports in key training sessions or specific training periods, as a quantitative tool to monitor training load and as a parameter to improve planning during the competitive season.

In the present study, the hypothesis that the hormonal GH/IGF-I axis concentrations present biphasic behavior during a training season $[3,5,6]$ was not observed, and there was no decrease in hormone concentrations, which could indicate a catabolic stage for further increases in concentrations as a chronic adaptation. Apparently, the hormonal GH/IGF-I axis concentrations may vary during the training period and these changes are related to the intensity of training phases and not

Table 5: Total calories intake obtained by the diet recall (R24h) at the 3 time points.

\begin{tabular}{|l|l|l|l|}
\hline & $\mathbf{1}$ & $\mathbf{2}$ & $\mathbf{3}$ \\
\hline R24h (Kcal) & $2669.69 \pm 358.64$ & $2681.23 \pm 350.53$ & $2670.78 \pm 354.12$ \\
\hline
\end{tabular}

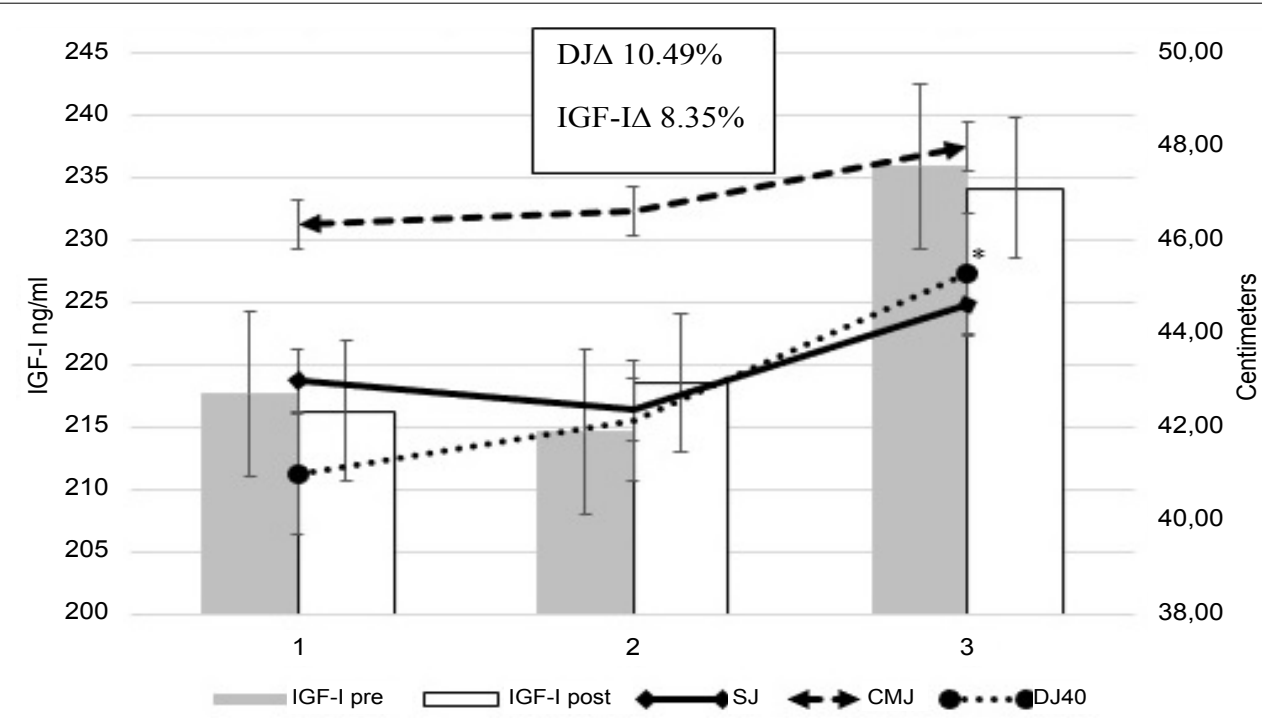

Figure 1: IGF-I pre and post training mean values and Squat Jump (SJ), Counter Movement Jump (CMJ) and $40 \mathrm{~cm}$ Drop Jump (DJ40) results throughout the training season.

*Statistical difference to A1 $(p<0.05)$. 
simply the length of time, as had previously been postulated.

According to Eliakin, et al. [19] the results of IGF-I concentrations are correlated positively with self-judgment of fitness in adolescent athletes of the Israel handball selection and data from Rosendal, et al. [20] corroborate the idea that IGF-I and IGFBP-3 concentrations are associated with the individual fitness level.

Tourinho Filho, et al. [21] analyzed the effects of a swimming training period on IGF-I and IGFBP-3 concentrations in swimmers aged 16 to 19 years. The authors found that IGF-I was sensitive to the acute and chronic effects of training and exhibited biphasic behavior during the period, with a catabolic phase marked by a reduction in IGF-I concentrations during the most intense period of training and an anabolic phase during the tapering phase.

Merji, et al. [22] observed no changes in IGF-I levels analyzed in soccer players during a competition season. The researchers also found higher levels of $\mathrm{GH}$ at the beginning of the season compared to the middle and end of the season. According to the authors, sports whose physical training planning was not aimed at a specific time of the season or a specific competition, and training intensity was maintained relatively constant during the season, changes could not be observed in IGF-I and IGFBP-3 concentrations.

Bogin, et al. [23] observed in different university team sports that in winners IGF-I baseline levels were $11 \%$ higher than in losers. It seems that IGF-I levels integrate the multiple genetic, nutritional, social and emotional complex that can possibly regulates athletic performance and, for the authors is the baseline levels of IGF-I and not the change in IGF-I levels during the competition that may contribute to winning. Based on this Eliakin and Nemet [24] suggested a novel cycle: Both single practice and prolonged training increase IGF-I levels, which could increase, probably, the chance of win. In this study an upward trend in IGF-I levels was observed in A3 and coincides with greater team results.

According to Ernst and Simon [25], exogenous administration to increase IGF-I concentrations had the ability to significantly improve results in athletic events requiring strength and speed, as in volleyball. This suggests that increased IGF-I concentrations at specific time points of the competitive season could be an indicator of better physical condition for competition, as was found at time point $A 3$ in this study.

The feeding state analysis through food recall $\mathrm{R} 24 \mathrm{~h}$ was not able to identify differences between the collection times. In addition, the R24h showed that athletes were not in a state of malnutrition or severe dietary restrictions which, according to Roemmich and Sinning [26], Nemet, et al. [27] and Nindl, et al. [28] has the potential of suppressing the IGF-I concentration.

In the present study, the typical volleyball training session did not reach the necessary intensity to cause significant changes in concentrations of IGF-I and IGFBP-3. Thereby the IGF-I e IGFBP-3 did not prove to be an adequate marker of training status in professional volleyball players.
This research did not receive any specific grant from funding agencies in the public, commercial, or not-for-profit sectors.

The author(s) have no conflicts of interest relevant to this article.

\section{References}

1. CD Martinelli Jr, RJ Custodio, MH Aguiar-Oliveira (2008) Physiology axis of GH/IGF-1. Arq Bras Endocrinol Metab 52: 717-725.

2. Eliakim A, JA Brasel, S Mohan, et al. (1996) Physical fitness, endurance training, and the growth hormone-insulin-like growth factor I system in adolescent females. J Clin Endocrinol Metab 81: 3986-3992.

3. Eliakim A, JA Brasel, S Mohan, et al. (1998) Increased physical activity and the growth hormone insulin-like growth factor-I axis in adolescent males. Am J Physiol 275: 308-314.

4. Nemet D, Y Oh, HS Kim, et al. (2002) Effect of intense exercise on inflammatory cytokines and growth mediators in adolescents boys. Pediatrics 110: 681-689.

5. KR Rarick, MA Pikosky, A Grediagin, et al. (2007) Energy flux, more than energy balance, protein intake, or fitness level, influences insulin-like growth factor I system responses during 7 days of increased physical activity. J Appl Physiol (1985) 103: 16131621.

6. Eliakim A, D Nemet (2010) Exercise training, physical fitness and the growth hormone-Insulin-Like growth factor-1 axis and cytokine balance. In: J Jürimäe, AP Hills, T Jürimäe, Cytokines, growth mediators and physical activity in children during puberty. Med Sport Sci, Basel, Karger 55: 128-140.

7. D Nemet, AM Pontello, C Rose-Gottron, et al. (2004) Cytokines and growth factors during and after a wrestling season in adolescent boys. Med Sci Sports Exerc 36: 794-800.

8. Eliakim A, D Nemet (2013) Exercise and the GH/IGF-1 axis. In: N Constantini, AC Hackney, ( $2^{\text {nd }}$ edn). Endocrinology of Physical activity and sport. Springer Science + Business Media, New York, 69-83.

9. Bosco C, P Luhtanen, P Komi (1983) A simple method for measurement of mechanical power in jumping. Eur J Appl Physiol Occup Physiol 50: 273-282.

10. Markwick WJ, Bird SP, Tufano JJ, et al. (2015) The intraday reliability of the reactive strength index calculated from a drop jump in professional men's basketball. Int J Sports Physiol Perform 10: 482-488.

11. Bosco C, Belli A, Astrua M, et al. (1995) A dynamometer for evaluation of dynamic muscle work. Eur J Appl Physiol Occup Physiol 70: 379-386.

12. (2013) Instituto Brasileiro De Geografia E Estatística (IBGE). Pesquisa Nacional de Saúde. Manual de Antropometria. Rio de Janeiro.

13. EM Evans, DA Rowe, MM Misic, et al. (2005) Skinfold prediction equation for athletes developed using a four-component model. Med Sci Sports Exerc 37: 2006-2011.

14. DP Guedes (1994) Composição corporal: Princípios técnicas e aplicações. ( $2^{\text {nd }}$ edn), Londrina, APEF.

15. Eliakin A, S Portal, Z Zadik, et al. (2013) Training reduces metabolic and inflammatory response to a single practice in female volleyball players. J Strength Cond Res 27: 3110-3115. 
16. WJ Kraemer, FS Harman, NH Vos, et al. (2000) Effects of exercise and alkalosis on serum insuline-like growth fator I and IGF-binding proteins. Can J Appl Physiol 25: 127-138.

17. Eliakin A, S Portal, Z Zadik, et al. (2009) The effect of a volleyball practice on anabolic hormones and inflammatory markers in elite male and female adolescent players. J Strength Cond Res 23: 1553-1559.

18. Nemet D, S Portal, Z Zadik, et al. (2012) Training increases anabolic response and reduces inflammatory response to a single practice in elite male adolescent volleyball players. J Pediatr Endocrinol Metab 25: 875-880.

19. Eliakin A, D Nemet, S Bar-Sela, et al. (2002) Changes in circulation IGF-1 and their correlation with self-assesment and fitness among elite athletes. Int J Sports Med 23: 600-603.

20. L Rosendal, L Henning, A Flyvbjerg, et al. (2002) Physical capacity influences the response of insulin-like growth factor and its binding proteins to training. J Appl Physiol (1985) 93: 1669-1675.

21. H Tourinho Filho, M Pires, EF Puggina, et al. (2017) Serum IGF-I, IGFBP-3 and ALS concentrations and physical performancein young swimmers during a training season. Growth Horm IGF Res 32: $49-54$

22. S Mejri, F Bchir, MC Ben Rayana, et al. (2005) Effect of training on GH and IGF-I responses to a submaximal exercise in football players. Eur J Appl Physiol 95: 496-503.

23. Bogin B, M Hermanussen, WF Blum, et al. (2015) Sex, sport, IGF1 and the community effect in height hypothesis. Int J Environ Res Public Health 12: 4816-4832.

24. Eliakim A, D Nemet (2020) Exercise and the GH-IGF-I axis. In: AC Hackney, NW Constantini ( $3^{\text {rd }}$ edn). Endocrinology of Physical Activity and Sports. Humana Press, New York, 71-84.

25. S Ernst, P Simon (2013) A quantitative approach for assessing significant improvements in elite sprint performance: Has IGF-1 entered the arena? Drug Test Anal 5: 384-389.

26. JN Roemmich, WE Sinning (1997) Weight loss and wrestling training: Effects on growth-related hormones. J Appl Physiol 82: 1760-1764.

27. Nemet D, CM Rose-Gottron, PJ Mills, et al. (2003) Effect of water polo practice on cytokines, growth mediators, and leukocytes in girls. Med Sci Sports Exerc 35: 356-363.

28. BC Nindl, JA Alemany, MD Kellog, et al. (2007) Utility of circulating IGF-I as a biomarker for assessing body composition changes in men during periods of high physical activity superimposed upon energy and sleep restriction. J Appl Physiol 103: 340-346.

DOI: $10.36959 / 987 / 251$

Copyright: (c) 2020 Pisa MF, et al. This is an open-access article distributed under the terms of the Creative Commons Attribution License, which permits unrestricted use, distribution, and reproduction in any medium, provided the original author and source are credited. 